\title{
Effects of drug relief hospital-based AIDS educational methods on drug users
}

\author{
Xiang Ping $\mathrm{LI}^{1}$, Shun Zhen XIAO ${ }^{1, *}$, Qiao Qin $\mathrm{WAN}^{1}$, Sen $\mathrm{Lin} \mathrm{SONG}^{2}$, Yan Xia TENG ${ }^{2}$ \\ ${ }^{1}$ School of Nursing, Peking University, Beijing 100083, China \\ ${ }^{2}$ Department of Psychiatry, DaXing Hospital, Beijing Drug Abuse Treatment Center, Beijing 102600, China
}

\begin{abstract}
The objective of this study is to explore a potentially effective training method for the hospital professionals to educate drug users and to enhance their knowledge of HIV infection. One hundred and sixty one subjects, who came from 13 different provinces and were admitted in a drug relief hospital in Beijing, were recruited for this study. The average age of these subjects was $35.21 \pm 6.24$ year old. The average numbers of years for drug addiction were 7 years, and the average numbers of drug relief treatment received in the past was 5.5 times. The level of AIDS knowledge of these subjects, including pathogenic factors, source of infection, route of transmission and preventive measures, were evaluated before and after receiving the AIDS educational training to these drug users. Our results showed that there was a statistically significant increase $(\mathrm{P}<0.01)$ in the knowledge of HIV infection and prevention among these subjects. Positive attitude and behavioral tendencies toward HIV prevention were also improved. Therefore, it is imperative for the medical professionals to incorporate AIDS education into drug relief treatment to achieve the maximum effect on the knowledge of AIDS and improvement of positive attitudes and behaviors toward HIV prevention among drug users.
\end{abstract}

Keywords: HIV infection; drug users; educational methods; attitude and behavirs.

\section{INTRODUCTION}

Today, Acquired Immunodeficiency Syndrome (AIDS) has become a public health and social problem that has caused significant harm to the survival and development of humanbeings. Route of transmission of Human Immunodeficiency Virus (HIV) is still predominantly through shared syringes by drug abusers. Currently, there is no efficient medicine, treatment or educational method to prevent HIV transmission in China. Intervention is probably the best "vaccine" [1]. Despite reinforced policies on education and intervention on AIDS carried out by the Chinese government; supporting measures are still lacking in many important areas, especially to the population that is difficult to reach (i.e., drug users, homosexuals

\footnotetext{
*Correspondence: Shun Zhen XIAO

School of Nursing, Peking University. 38 Xueyuan Road, Haidian District, Bejing 100083, China;

Tel: 011-86-10-8280-2106; Fax: 011-86-10-6201-5681;

E-mail: szxaio@bjmu.edu.cn
}

and unlicensed prostitutes). Since October 2004, we started to explore the potential effect of AIDS education on drug users. We first evaluated the situation of AIDS awareness and knowledge among the drug users in a drug relief hospital in Beijing. Our goal was to develop a potentially effective educational method for the medical professionals to improve treatment efficacy by incorporating an AIDS education program.

\section{MATERILAS AND METHODS}

\section{Subjects}

Candidates were selected among patients of a drug relief hospital in Beijing. 161 participants from 13 different provinces received preeducation investigation, and among them, there were 124 male (77. $1 \%), 37$ female (22.9\%); the average age was $35.21 \pm 6.24$ year old. The average numbers of years for drug addiction were 7 years, and the average number of received treatment on drug relief was 5.5 times.

\section{Content of education}

Knowledge of pathogenic factors, source of infection, route of transmission and preventive measures, etc. 


\section{Methods and procedures}

(1) In order for the medical staff to have a comprehensive understanding of AIDS knowledge, seminars were held in this hospital. (2) Training seminars were provided for AIDS knowledge to the education staff. The training method was based on participation, including small group teaching, group discussion, and on-site demonstration of education methods and contents of AIDS Education. (3) The objective of education of AIDS knowledge: Volunteers who are currently under treatment of drug treatment. (4) Methods of education: Face to face tutoring and answering questions. Education took place during the following period; 1-2 days once the patient was hospitalized and 3-5 days in the middle of treatment. Each questionnaire regarding AIDS knowledge would be completed by the selected patients on the first day and 1 day prior to leaving the hospital. (5) Grading method: there were 38 AIDS knowledge related questions. The total score was 38 . One point was given to each correct answer; zero points were given to each incorrect answer and to questions left blank.

\section{Statistics}

Data was entered into computer. SPSS10.0 software was used for statistics. Methods include describable statistical analysis, paired $t$-test and $\chi^{2}$-test.

\section{RESULTS}

Time and frequency of Education: the frequency of education was twice. The approximate time was about 30 minutes each. On average, the interval between two sessions was about $3.00 \pm 1.57$ days. There was no denial or uncooperative action during the education. 161 questionnaires were sent out, 160 valid questionnaires were collected. Recovery percentage of the questionnaires was $99.4 \%$.

High risk factors in spreading HIV/AIDS exist among drug users: Among 161 drug users who received pre-education base line investigation, $85.5 \%$ had only one sexual companion; $14.5 \%$ had more than one sexual companions; $7.7 \%$ used a condom during sexual intercourse, $48.1 \%$ used a condom during sexual intercourse occasionally, $44.2 \%$ did not use a condom during sexual intercourse; $14.3 \%$ used drugs by intramuscular/subcutaneous injection, $33.5 \%$ used drugs by intravenous injection. Among them, $83.6 \%$ have never shared the same syringe with others while using drugs, $13.2 \%$ shared the same syringe with others while using drugs occasionally, 3.3\% often shared the same syringe with others while using drugs.

Changes in AIDS awareness before and after receiving information: After: the total score (among selected drug users) of AIDS basic knowledge increased from 26.81 \pm 5.57 points (before education) to $31.80 \pm 3.78$ points (after education). The increase rate was 4.99 points This demonstrated a significant difference before / after receiving education ( $t=10.372, P<0.01)$. Among the rest, the rate of correct answers in 15 AIDS related questions were over $90 \%$ after receiving information. Some of the questions reached $95 \%$ rate of correct answers. Tab. 1 shows detailed information.

Changes in attitude towards AIDS (before/after education): The difference in attitude towards AIDS among the drug users (before/after education) mainly exhibited in whether they had the risk of infecting AIDS themselves. Before education, 34 (21.3\%) regarded themselves at the risk of infecting AIDS; after education, this figure was 59 $(36.9 \%)$. The difference demonstrated was very significant $\left(\chi^{2}=9.47, P<0.01\right)$. After receiving the AIDS education, $146(91.3 \%)$ expressed that they would circulate this information to their family members.

Self awareness in risk of infecting HIV/AIDS: after education, $59(36.6 \%)$ were aware they had the risk of infecting HIV/AIDS, increased by $14.9 \%$. This demonstrated a significant difference $\left(\chi^{2}=8.90, P<0.01\right)$.

Tendencies in behavior related to AIDS (after education): During this study, the tendencies of behavior primarily focused in the following areas: whether the candidates would take the AIDS test voluntarily, whether they used condoms during sexual intercourse and whether they shared syringes while drug use. For detail results, please refer to Tab. 2.

\section{DISCUSSIONS}

The number of registered drug users has increased to approximately 860,000 in 2000 . Among them, about $17 \%$

Tab. 1 Items in AIDS knowledge that rate of correct answer reached 95\% $(\mathrm{n}=160)$

\begin{tabular}{lllrr}
\hline Contents (AIDS related knowledge) & $\begin{array}{l}\text { Score before } \\
\text { education (\%) }\end{array}$ & $\begin{array}{l}\text { Score after } \\
\text { education (\%) }\end{array}$ & $\chi^{2}$ & $P$ \\
\hline Sexual intercourse will spread AIDS & $137(85.6)$ & $156(97.5)$ & 13.56 & $<0.01$ \\
Sharing syringe will spread AIDS & $131(81.9)$ & $156(97.5)$ & 21.12 & $<0.01$ \\
Sharing needling instrument to inject drugs will spread AIDS & $148(92.5)$ & $159(99.4)$ & 9.70 & $<0.01$ \\
Will handshake, hug have the risk of infecting AIDS & $148(92.5)$ & $157(98.1)$ & 5.67 & $<0.05$ \\
Will sexual intercourse have the risk of infecting AIDS & $137(85.6)$ & $153(95.6)$ & 9.42 & $<0.01$ \\
\hline
\end{tabular}


Tab. 2 Comparison in tendencies of AIDS related behavior of volunteers in drug habits after education $(\mathrm{n}=160)$

\begin{tabular}{|c|c|c|c|c|c|}
\hline $\begin{array}{l}\text { AIDS related behavior } \\
\text { Contents }\end{array}$ & Answer & $\begin{array}{l}\text { Before education } \\
\text { case } \%\end{array}$ & $\begin{array}{c}\text { After education } \\
\text { case } \% \\
\end{array}$ & $\chi^{2}$ & $P$ \\
\hline Use condoms during & All the time & $12(7.7)$ & $55(35.0)$ & \multirow{3}{*}{59.79} & \multirow{3}{*}{$<0.01$} \\
\hline \multirow[t]{2}{*}{ Sexual intercourse } & Between times & $74(47.7)$ & $85(54.1)$ & & \\
\hline & Never & $69(44.5)$ & $17(10.8)$ & & \\
\hline Will share the same & Yes & $25(16.6)$ & $7(4.6)$ & \multirow{2}{*}{11.33} & \multirow{2}{*}{$<0.01$} \\
\hline Syringe & No & $126(83.4)$ & $144(95.4)$ & & \\
\hline \multirow[t]{2}{*}{ Receive AIDS test } & Agree & $83(52.9)$ & $102(63.8)$ & \multirow{2}{*}{4.63} & \multirow{2}{*}{0.032} \\
\hline & Disagree & $77(47.1)$ & $58(36.2)$ & & \\
\hline
\end{tabular}

were female (this number demonstrated a tendency of increase). Most of them were involved in both drug addiction and prostitution. This built a bridge between drug users and the general public [2]. To carry on an extensive and permanent educational program through multi-formalities and channels toward variant groups, especially high risk and weak areas, has become a critical problem that needs to be solved in preventing HIV/AIDS.

The education methods of this research enforced a positive effect on the drug users' knowledge, attitude and behavior tendencies towards AIDS.

Impacts of AIDS knowledge: after education, the percentage of correct answers in AIDS knowledge had a significant increase, especially regarding the questions of whether sexual contact and sharing syringes will cause AIDS. This data illustrates that the drug users lacked the awareness of the hazards surrounding them. After receiving the education, they paid great attention to this point. Through this education, knowledge may be effectively increased.

Impacts of attitude towards AIDS: This research indicated that after education, the individuals were more concerned about whether they had the risk of getting infected by HIV. After education, $91.3 \%$ of them were willing to pass on AIDS information to their family members and sexual companions. This indicates that education is essential for the drug users to establish a positive attitude. Furthermore, it may stimulate the promotion of AIDS knowledge to both the general public and drug users to a certain degree.

Impacts on tendencies of AIDS related high risk behavior: The result of this research indicates that after receiving education, the drug users tended to change positively in the following two crucial links; usage of condoms and avoid sharing syringes with others. This exemplifies that education may be in favor of increasing positive behaviors of drug users. It bears more positive connotation, especially for changing high risk related behaviors of the individuals.
The advantages of face-to-face personal education for drug users who are hospitalized in drug treatment hospitals are:

The ability to contact the targeted groups effectively: Drug users, being one of fringe groups in society, are difficult to approach and cooperate with. It is better to promote AIDS education in a drug treatment hospital. Furthermore, since the medical staff in the hospital demonstrated the effectiveness of the education, it was easier for them to approach the targeted group.

Being educational staff, the medical staff ensured the quality and consistency of the information: This research selected participants among volunteers with good interpersonal communication skills to attend the seminars for AIDS related knowledge. On one hand, it will reassure the quality and consistency of the education; and on the other hand, it will gain the patients' trust and confidence.

It may cover certain number of drug users within a short period of time: This research exhibits that there were 160 drug users who received education in AIDS knowledge within two months, which indicated educationfor a number of drug users can be carried out within a short period of time. In addition, the patients in this particular hospital who were receiving drug treatment were not only from the Beijing area, but also from 12 other provinces of China. This certainly represents the vast movement of drug users; however, it also indicated that this education method could be effectively applied to other areas.

Health Education may enhance the awareness of drug users in risks of AIDS infection: The findings indicate that the AIDS awareness and its risks were significantly improved after receiving the information. This can be illustrated by providing the comprehensive AIDS related knowledge given by the medical staff. The drug users can obtain this information, and therefore they can understand the concept of AIDS. This indicates that providing detailed education about AIDS can improve knowledge for drug users and aid them to behave responsibly.

Social meaning of this education method studies: Chang- 
ing of behavior is the ultimate objective and is the key of health education. Companion Dynamical Theory and Identical Consistency Theory [3] have provided rationale for social meanings for this education method. Companion Dynamical Theory states that people in society have convergence pressure. When someone's stance differs with others, then the change will be apt to the stance of colony. For instance, if sharing a syringe is considered unacceptable in the societal environment, then it will be difficult for individuals to do so. Therefore, it is imperative to provide education to drug users for AIDS prevention. As a result, the knowledge will help them to change their personal mentalities and behavior.

\section{ACKNOWLEDGMENT}

This work is supported in part by fund of the University of Illinois at Chicago AIDS International Training Research Program (AITRP).

\section{REFERENCES}

1 Yi ZENG, Hua XU. Educational Propaganda and intervention are the effective measures in containing outbreak of AIDS. Medical Tribune Journal, 2004, 25: 1-6.

2 Zun You WU. Risky behaviors related to AIDS and its intervention. China Epidemiology Journal, 2001, 22: 321-2.

3 Dong Bao YU, Jiang Ping SUN. Practice of Health Promotion Theory and its practice in prevention and Control of AIDS. Journal for China AIDS/STD Prevention and Control, 2002, 8: 315-7. 